\title{
Vesicular Formation of Trans-Ferulic Acid: an Efficient Approach to Improve the Radical Scavenging and Antimicrobial Properties
}

\author{
Anahita Rezaeiroshan ${ }^{1,2} \cdot$ Majid Saeedi $^{1} \cdot$ Katayoun Morteza-Semnani $^{3} \cdot$ Jafar Akbari $^{1}$. \\ Akbar Hedayatizadeh-Omran ${ }^{4} \cdot$ Hamidreza Goli $^{5} \cdot$ Ali Nokhodchi $^{6}$ (i)
}

Accepted: 7 February 2021 / Published online: 1 March 2021

(c) The Author(s) 2021

\begin{abstract}
Purposes Reactive oxygen species production is harmful to human's health. The presence of antioxidants in the body may help to diminish reactive oxygen species. Trans-ferulic acid is a good antioxidant, but its low water solubility excludes its utilization. The study aims to explore whether a vesicular drug delivery could be a way to overcome the poor absorption of trans-ferulic acid hence improving its antimicrobial efficiency and antioxidant effect.

Methods Niosomal vesicles containing the drug were prepared by film hydration method. The obtained vesicles were investigated in terms of morphology, size, entrapment efficiency, release behavior, cellular cytotoxicity, antioxidant, cellular protection study, and antimicrobial evaluations.

Results The optimized niosomal formulation had a particle size of $158.7 \mathrm{~nm}$ and entrapment efficiency of $21.64 \%$. The results showed that the optimized formulation containing $25 \mu \mathrm{M}$ of trans-ferulic acid could enhance the viability of human foreskin fibroblast HFF cell line against reactive oxygen species production. The minimum effective dose of the plain drug and the niosomal formulation against Staphylococcus aurous (ATCC 29213) was $750 \mu \mathrm{g} / \mathrm{mL}$ and $375 \mu \mathrm{g} / \mathrm{mL}$, respectively, and for Escherichia coli (ATCC 25922), it was $750 \mu \mathrm{g} / \mathrm{mL}$ and $187 / 5 \mu \mathrm{g} / \mathrm{mL}$, respectively. The formulation could also improve the minimum bactericidal concentration of the drug in Staphylococcus aurous, Escherichia coli, and Acinobacter baumannii (ATCC 19606).
\end{abstract}

Conclusion These results revealed an improvement in both antibacterial and antioxidant effects of the drug in the niosomal formulation.

Keywords Trans-ferulic acid $\cdot$ Vesicular delivery $\cdot$ ROS production $\cdot$ Antibacterial effect $\cdot$ In vitro release $\cdot$ Radical scavenging activity

Ali Nokhodchi

a.nokhodchi@sussex.ac.uk

Majid Saeedi

msaeedi@mazums.ac.ir

1 Pharmaceutical Sciences Research Centre, Hemoglobinopathy Institute, Mazandaran University of Medical Sciences, Sari, Iran

2 Department of Pharmaceutics, Faculty of Pharmacy, Mazandaran University of Medical Sciences, Sari, Iran

3 Department of Medicinal Chemistry, Faculty of Pharmacy, Mazandaran University of Medical Sciences, Sari, Iran

4 Gastrointestinal Cancer Research Center, Mazandaran University of Medical Sciences, Sari, Iran

5 Department of Medical Microbiology and Virology, Faculty of Medicine, Mazandaran University of Medical Sciences, Sari, Iran

6 Pharmaceutics Research Laboratory, School of Life Sciences, University of Sussex, Brighton BN1 9QJ, UK

\section{Introduction}

Ferulic acid (4-hydroxy,3-methoxy cinnamic acid) is an organic phytochemical and a derivative of hydroxycinnamic acids which is widely distributed in nature and is available in many foods of human dietary such as tomato, eggplant, rice, banana, wheat, etc. Transferulic acid (TFA) has many pharmacological properties including antioxidant, antimicrobial, antifungal, and anti-inflammation. TFA poses radical scavenging activity due to phenoxy radical. The methoxy and carboxylic acid moieties can form a stabilized resonance. The carboxylic group of the drug performs as an anchor of TFA which helps to bind to the lipid bilayers and prevents lipid peroxidation. It is clear that the electron-donating substituents can enhance the antioxidant activity of TFA 
[1]. This compound also possesses cytoprotective effects as a result of its capability to hinder the genes expression related to cell apoptosis [2].

Reactive oxygen species (ROS) are defined as chemicals that contain oxygen in their structure. ROS production in the body may develop damages to the cells. It is reported that the initial cause of various chronic diseases such as cancer, neurodegenerative diseases, inflammatory and cardiovascular diseases may begin by the accumulation of these species in the body. The regular usage of antioxidants can help to diminish these species in tissues. Dietary compounds are a rich source of antioxidants which can increase cellular defense against oxidation damage. Polyphenols as natural antioxidants are considered to be very useful due to their numerous biological benefits such as free radical scavenging activity, the ability to chelate metals and attenuating the enzymatic activity [3]. It has been shown that TFA is an antioxidant and it may help to stop the cytotoxicity of chemotherapy drugs [4]. The important advantage of dietary antioxidants is their ability to defend against oxidative damages induced by the production of ROS [5].

Furthermore, it is reported that TFA can elevate the expression levels of antioxidant enzymes, superoxide dismutase (SOD) and catalase. The number and position of the phenolic groups may influence the antioxidant activity, so different phenolic compounds reveal different biological functions [6]. TFA has antibacterial property as well as antioxidant feature. In the previous study, researchers have proved the ability of herbal compounds to be utilized as adjuvants together with antibacterial drugs [7].

Application of nanocarriers attracted a lot of interests these days due to the benefits over conventional drug delivery system. Nano-drug delivery can increase the bioavailability of drugs with fast first-pass effect, enhances the pharmacokinetic profile of drugs. Niosomes have a vesicular structure formed by the association of nonionic surfactants. The ability of these vesicular bilayers to encapsulate hydrophilic and hydrophobic drugs, controlling the release of the drug and avoid dose dumping which decreases the compliance of the patient and target delivery of the drug, are the major advantages of niosomes which made theme unique for further studies [8]. As TFA has poor solubility in water, therefore, its bioavailability through oral administration is poor. It has been shown that niosomes can encapsulate both hydrophilic and hydrophobic compounds, so the authors believed that niosomes could be a great choice for TFA to enhance its performance [9]. Furthermore, the radical scavenging and antimicrobial potential of TFA could be enhanced if the drug is encapsulated in the bilayer structure of niosomes.

\section{Material}

Trans-ferulic acid (TFA) with $99 \%$ purity was purchased from Sigma-Aldrich (St. Louis, MO, USA). Sorbitan monostearate (Span 60), polysorbate 60 (Tween 60) were purchased from Merck KGaA (Germany). Cholesterol was obtained from Solar Bio Chemicals Reagent Company (China). Deionized water (DW) was obtained from Human Ultra-Pure System (Human Corp, Korea). All chemicals were of analytical grade and purchased from Merck KGaA (Darmstadt, Germany).

\section{Methods}

\section{Niosome Preparation and Characterization}

Niosomal vesicles were prepared by film hydration method, which is explained in our previous paper [10]. As the aim of the current study was to investigate the antioxidant effect of the obtained niosomes, therefore, further details of making niosomes were not reported here. Briefly, appropriate amounts of drug, cholesterol, and Span 60 were dissolved in chloroform and methanol $(1: 1 \mathrm{v} / \mathrm{v})$; then, the mixture was evaporated to form a thin layer around the round bottom flask (Table 1). Tween 60 and water were then added to hydrate the mixture within $45 \mathrm{~min}$ at $73^{\circ} \mathrm{C}$ and $250 \mathrm{rpm}$ to obtain niosomes.

In brief, niosome formulation $(1.5 \mathrm{~mL})$ was centrifuged by using a refrigerated ultracentrifuge (Sigma, Germany). The supernatant was removed from the centrifuged tube with a syringe and the amount of drug was determined by a standard curve (Jasco, v630,

Table 1 The composition of the formulations

\begin{tabular}{lcc}
\hline Run & HLB value & Cholesterol $(\mathrm{mg})$ \\
\hline F1 & 9.00 & 90.00 \\
F2 & 9.00 & 90.00 \\
F3 & 9.00 & 90.00 \\
F4 & 4.76 & 90.00 \\
F5 & 12.00 & 30.00 \\
F6 & 13.24 & 90.00 \\
F7 & 6.00 & 150.00 \\
F8 & 9.00 & 90.00 \\
F9 & 9.00 & 90.00 \\
F10 & 12.00 & 150.00 \\
F11 & 9.00 & 174.85 \\
F12 & 6.00 & 30.00 \\
F13 & 9.00 & 5.15 \\
\hline
\end{tabular}


spectrophotometer, Japan), at $320 \mathrm{~nm}$. Since the total amount of the drug is known, then the following equation was used to calculate the EE\%.

Drug entrapment $\%=$ (total drug - drug in supernatant $) /$ (total drug) $\times 100$.

\section{Morphology Evaluation}

Analyzing the morphology of the formulation was carried out by scanning electron microscopy (SEM). The samples were coated with gold and the morphology of the samples was analyzed by the apparatus at different magnifications (SSX-550 Superscan, Shimadzu, Kyoto, Japan) [11].

\section{Release Behavior Study}

In vitro release was carried out by dialysis bag method [12]. In brief, $3 \mathrm{~mL}$ of the formulation and the drug suspension were transferred to a $50-\mathrm{mL}$ falcon tube containing $40 \mathrm{~mL}$ of saline and 5\% PEG 400 . The falcon tubes were kept at $37 \pm 0.5{ }^{\circ} \mathrm{C}$ and stirred at $90 \mathrm{rpm}$ on a shaker incubator. At appropriate time intervals $(0.5$, $1,2,4,6,8,10$, and $24 \mathrm{~h}$ ), $2 \mathrm{~mL}$ of the samples were withdrawn and replaced with the same amount of fresh medium. The absorbance of the withdrawn samples was then determined by UV spectroscopy at a wavelength of $320 \mathrm{~nm}$. In order to study the mechanism of drug release from the vesicles, Kinet DS software version 3.0 (Jagiellonian University Medical College, Poland) was employed. The drug release data were fitted to various kinetic models namely zero-order, first-order, Higuchi and Korsmeyer-Peppas. The determination coefficient $\left(r^{2}\right)$ for all models was used to select the best-fitting model.

\section{In vitro Cell Culture-based Assays}

\section{Cell Culture}

The (HFF PI6) Human foreskin fibroblasts cell line) NCBI Code: C170) and L929 murine)NCBI code: C161) fibroblast cell line was cultivated in $75 \mathrm{~cm}^{2}$ flasks including RPMI 1640 medium supplemented with $2 \mathrm{mM} \mathrm{L}$-glutamine, $24 \mathrm{mM} \mathrm{NaHCO}_{3}, 10 \%$ fetal bovine serum, penicillin (100 U/ $\mathrm{mL})$ and streptomycin $(100 \mu \mathrm{g} / \mathrm{mL})$ under $5 \% \mathrm{CO}_{2}$ at $37{ }^{\circ} \mathrm{C}$. All the results were reported as the mean of three individual experiments.

\section{Cytotoxicity Assay}

The MTT [3-(4, 5-dimethylthiazol-2-yl)-2, 5-diphenyltetrazolium bromide] assay was performed for detection of the cell viability. MTT powder is a water-soluble yellow tetrazolium salt which gets into a redox reaction by the existence of the mitochondrial succinate dehydrogenase enzyme in the living cells and gets into a transformation water-insoluble formazan dye. In brief, the HFF and L929 cells (with the density of $10 \times 10^{3}$ and $15 \times 10^{3}$, respectively) were seeded in 96-well plates. After overnight adhesion, cells were treated with different concentrations of TFA and TFA niosome at $37{ }^{\circ} \mathrm{C}$ in the medium for 24 and $48 \mathrm{~h}$. After passing some time, MTT solution $(5 \mathrm{mg} / \mathrm{mL}$ in PBS) was added to the plates and incubated for $3 \mathrm{~h}$. The supernatant was then removed and $200 \mu \mathrm{L}$ of dimethyl sulfoxide (DMSO) solution was added to each of the wells to break down the cell membrane and remove the formazan dyes. Each plate was put on a shaker for about $20 \mathrm{~min}$. Finally, the absorbance was evaluated at $\chi=570 \mathrm{~nm}$ with a microplate reader (Synergy H1 hybrid multi-mode microplate reader Bio-Tek Instruments, Winooski, VT, USA) [13].

\section{Cytoprotection Assay}

Human HFF fibroblast cells were maintained in RPMI medium under the conditions previously mentioned. Cells were cultured at passage 15 on a 96 -well plate with the densities used in the MTT assay and after an overnight, 25 and $50 \mu \mathrm{M}$ of TFA and TFA niosome were added to the cells and incubated for $24 \mathrm{~h}$. To induce oxidative stress, $300 \mu \mathrm{M}$ of $\mathrm{H}_{2} \mathrm{O}_{2}$ solution was added to each of the wells that were treated with plain TFA, noisome containing TFA and the positive control group $\left(\mathrm{H}_{2} \mathrm{O}_{2}\right.$ alone $)$ was incubated for $4 \mathrm{~h}$. MTT assay was used to evaluate the protective effect of these interventions by determining cell viability. Briefly, $20 \mu \mathrm{L}$ of MTT solution was added to the plates and incubated for $3 \mathrm{~h}$ then DMSO was added and the plates were shaken on the shaker for $20 \mathrm{~min}$ followed by the absorbance measurement by a microplate reader at $570 \mathrm{~nm}$ [14].

\section{Assay for Intracellular Reactive Oxygen Species Generation}

The content of cellular ROS was measured by a ROSsensitive fluorescence marker, $2^{\prime}, 7^{\prime}$-dichlorofluorescein diacetate (DCFH-DA). ROS inhibition was measured by TFA niosomes. In brief, $3 \times 10^{4} \mathrm{HFF}$ cells were cultured 
in each of the 96 well black plates and after passing an overnight, the cells were treated by TFA and niosomal formulation of TFA at the concentration of 25 and $50 \mu \mathrm{M}$ followed by incubation at $37{ }^{\circ} \mathrm{C}$ with $5 \% \mathrm{CO}_{2}$. The cells were then treated with $\mathrm{H}_{2} \mathrm{O}_{2}$ with a concentration of $300 \mu \mathrm{M}$ for $4 \mathrm{~h}$ to induce oxidative stress. After washing the cells with PBS, cells were incubated with DCFHDA $(25 \mu \mathrm{M})$ for $45 \mathrm{~min}$ in phenol-free culture medium at $37{ }^{\circ} \mathrm{C}$ in the dark. Fluorescence intensity was assayed at an excitation wavelength of $485 \mathrm{~nm}$ and an emission wavelength of $535 \mathrm{~nm}$ using a Synergy H1 hybrid multimode microplate reader [15].

\section{Antibacterial Activity of the Formulation}

To evaluate the MIC and minimum bactericidal concentration (MBC) of the formulation, the microbroth dilution method was assessed according to the Clinical and Laboratory Standards Institute (CLSI) [16]. A 96-well plate was used in the test, while a series of twofold dilutions of the drug and the formulation were prepared in Mueller-Hinton broth (Merck, Germany). Then, the bacterial suspension $\left(\sim 10^{5} \mathrm{cfu} / \mathrm{mL}\right)$ was added to all wells in the plates, except for the positive and negative control wells, followed by incubation at $37^{\circ} \mathrm{C}$ for $24 \mathrm{~h}$. In this experiment, a well with drug and broth medium was used as a negative control, while the combination of bacterial suspension and broth medium was used as a positive control. The well without microbial growth and turbidity was considered as the MIC rate of the drug against the tested bacteria $(\mu \mathrm{g} / \mathrm{mL})[17]$.

\section{Bacteria}

Staphylococcus aureus (ATCC 29213), Escherichia coli (ATCC 25922), Enterococcus faecalis (ATCC 29212) Pseudomonas aeruginosa (ATCC27853), Acinetobacter baumannii (ATCC19606), Klebsiella pneumonia (ATCC 700603), and Proteus mirabilis (ATCC 25933) were used to evaluate the antimicrobial activity of the formulation in this study. These strains were obtained from the department of microbiology, School of Medicine, Mazandaran University of Medical Sciences, Sari, Iran.

\section{Statistical Analysis}

SPSS software version 16 was used to analyze the data statistically. One-way analysis of variance (ANOVA) followed by Tukey's post hoc test were used to measure mean cell proliferation and antioxidant effect. IC50 levels were calculated for different cell lines using aatbio web software. $P$ value of less than 0.05 was considered as the level of significance.

\section{Results}

\section{TFA Niosome Characterization and Morphology Evaluation}

The results showed that the size and the entrapment efficiency of the optimized formulation were $158.7 \mathrm{~nm}$ and $21.64 \%$, respectively. Tween 60 has larger hydrophilic head group and higher HLB value (14.9) in comparison to Span 60 (4.7) which is the main cause to form a suitable molecular geometry for the bilayer structures of niosomes. Cholesterol is used to avoid the probable leakage through the vesicle and it is usually known as the hardener of the structure. For further information, the authors suggest readers refer to our published paper [10]. SEM images are shown in Fig. 1. The SEM image shows that the vesicles are well distributed and they are in nano-sized range $(90.1 \pm 9.75 \mathrm{~nm}$ to $304.2 \pm 6.47 \mathrm{~nm})$.

\section{In vitro Release}

The in vitro release profile of TFA niosome and drug suspension are shown in Fig. 2. The figure shows that the niosomal formulation could be released slower than the drug suspension. The results showed that these two drug release profiles are statistically significant from each other $(P<0.05)$. Various kinetic models (firstorder, Higuchi, zero-order, and Korsmeyer-Peppas) were employed and the best model was selected based on the highest $R^{2}$ and the lowest residual square mean error (RSME). The results are shown in Table 2 . Korsmeyer-Peppas model was the best model to fit the drug release data for both the TFA niosomal formulation and the drug suspension.

\section{Cytotoxic Effect Evaluation}

Cytotoxicity analysis is reported in Fig. 3. The figure showed that different doses of niosomes containing TFA showed different toxicity on normal HFF cells. The 
Fig. 1 Image of TFA niosome through scanning electron microscope

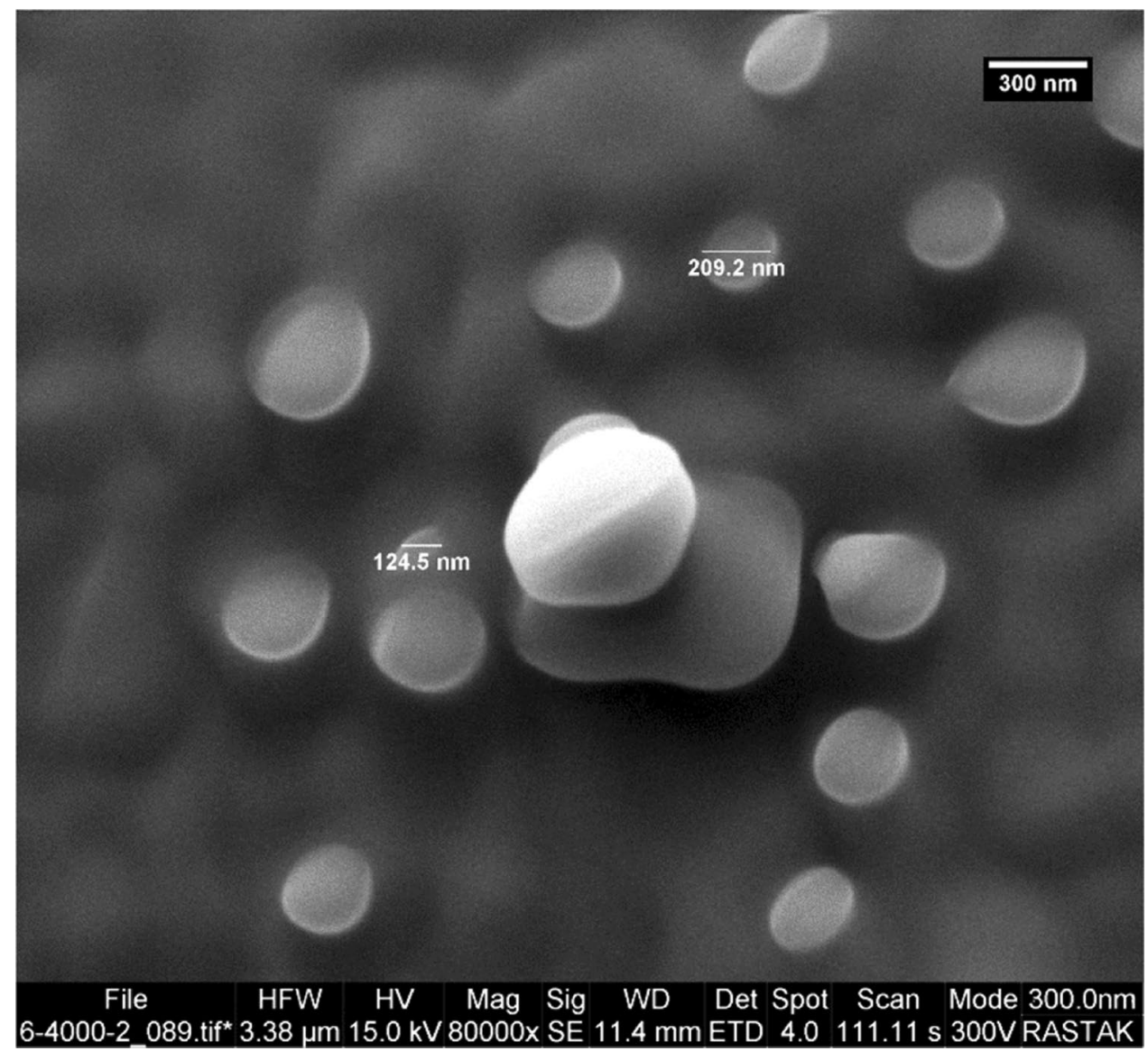

effect of dose on the toxicity of HFF cells was significant $(P<0.05)$ in 24 -h treatment but in 48 -h treatment, there was no significant difference $(P>0.05)$. This was not the case when normal L929 cells were used as the difference was significant at both treatment times $(P<0.05)$. The IC50 value on HFF and L929 cells at

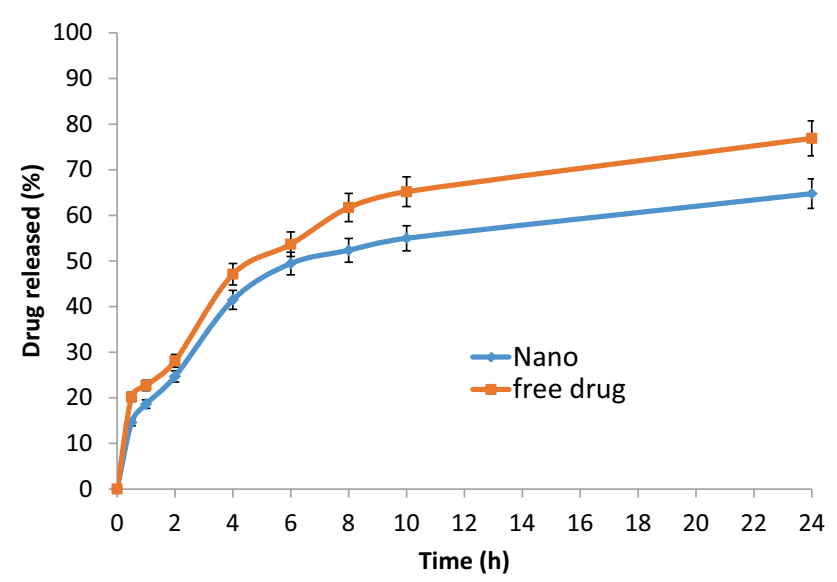

Fig. 2 The release profile of TFA (free drug) and TFA niosomal (Nano) formulation 24-h are $577.62 \mu \mathrm{M}$ and $255.62 \mu \mathrm{M}$, respectively. IC50 indicates a concentration of the drug that inhibits $50 \%$ of cell growth. The higher the IC50 value, the safer the drug is. Figure 3 shows that the cytotoxicity effect on HFF cells was lower in $24 \mathrm{~h}$ when niosomal formulation was used.

Table 2 Determination coefficient $\left(R^{2}\right)$ and residual square mean error (RSME) for various drug release kinetic models

\begin{tabular}{llrllc}
\hline & \multicolumn{2}{l}{ TFA suspension } & & \multicolumn{2}{l}{ Optimized TFA } \\
& & & & \multicolumn{2}{l}{ Formulation } \\
\cline { 2 - 3 } \cline { 5 - 6 } & $R^{2}$ & RSME* $^{2}$ & & $R^{2}$ & RSME \\
\hline Higuchi & 0.921 & 15.09 & & 0.89 & 16.60 \\
First order & 0.874 & 27.93 & & 0.796 & 28.83 \\
Korsmeyer-Peppas* & 0.929 & 9.76 & & 0.982 & 6.00 \\
Zero order & 0.744 & 32.29 & & 0.699 & 31.1 \\
\hline
\end{tabular}

* $n$ value for Korsmeyer-Peppas model was 0.479 and 0.477 for niosomal formulation and drug suspension, respectively. This indicates the main mechanism of drug release is diffusion 
Fig. 3 A cell viability measured using MTT assay in 24 and 48 h. NF niosome ferulic acid, $\mathrm{F}$ ferulic acid
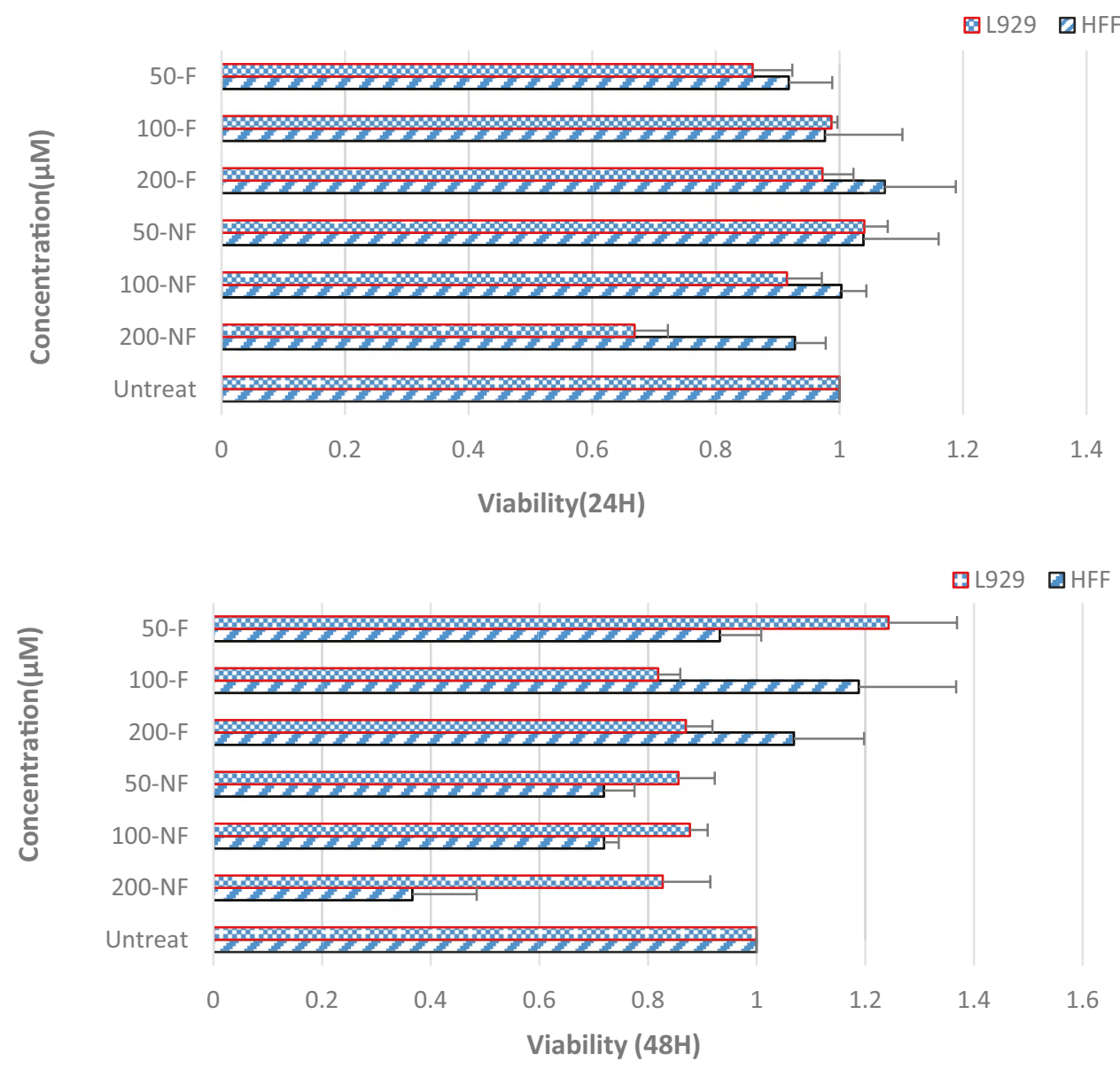

\section{Antioxidant Activity}

To determine the optimal dose of $\mathrm{H}_{2} \mathrm{O}_{2}$, HFF cells were treated with different concentrations of $\mathrm{H}_{2} \mathrm{O}_{2}$. According to the experiments, the cellular ROS content after treatment with $300 \mu \mathrm{M} \mathrm{H}_{2} \mathrm{O}_{2}$ was higher than other concentrations and this difference was statistically significant $(P<0.05)$. In the subsequent experiments, $300 \mu \mathrm{M}$ of $\mathrm{H}_{2} \mathrm{O}_{2}$ for $4 \mathrm{~h}$ was used to

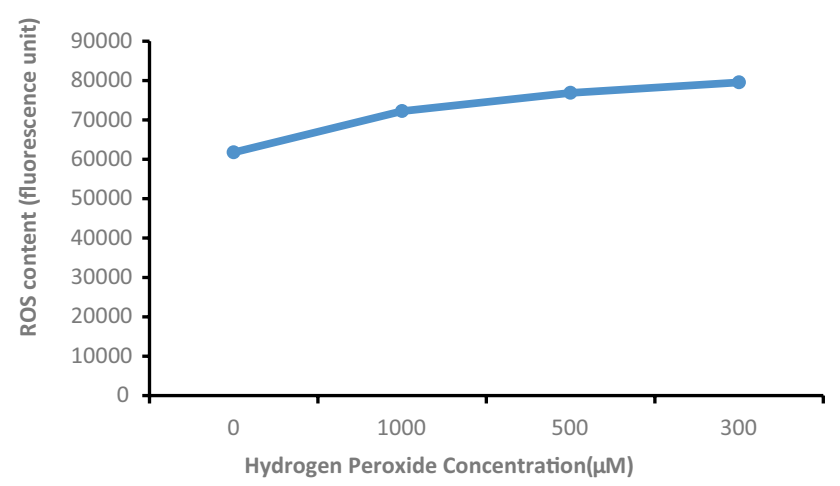

Fig. 4 Induction of oxidative stress by intracellular ROS production in $\mathrm{HFF}$ cells using $\mathrm{H}_{2} \mathrm{O}_{2}$ induce oxidative stress (Fig. 4). The decrease in ROS production after 24-h treatment of HFF cells with TFA and TFA niosomes is visible at 25 and $50 \mu \mathrm{M}$ concentrations (Fig. 5). This decrease in peroxide ions has been identified based on evidence of uptake of ROS-sensitive fluorescence markers called DCFDH. Cells treated with TFA niosomes at a concentration of $25 \mu \mathrm{M}$ had lower fluorescence than the control group $\left(\mathrm{H}_{2} \mathrm{O}_{2}\right.$ treatment), which was statistically significant $(P<0.05)$.

\section{Cellular Protection Study}

The protective role of TFA niosomes against hydrogen peroxideinduced oxidative stress was investigated. Cell viability was decreased $(P<0.05)$ by exposure to hydrogen peroxide in HFF cells. Niosomal formulation of TFA at $25 \mu \mathrm{M}$ increased the viability of HFF cell line, which was not statistically significant $(P>0.05)$. The results are shown in Fig. 6.

\section{Antimicrobial Results}

Antimicrobial activities of TFA and niosome encapsulated TFA against bacteria are presented in Table 3. Niosomal formulation showed better antibacterial activity against the tested bacteria compared to plain TFA. 
Fig. 5 Intracellular ROS production in HFF cells using DCFH-DA fluorescence

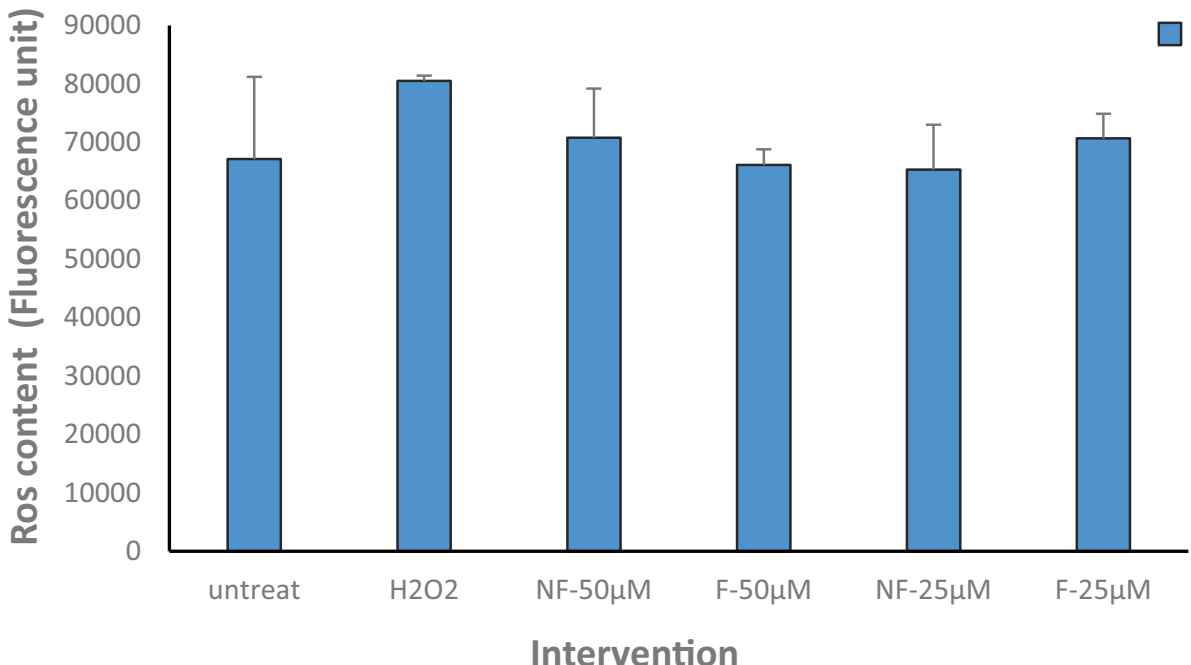

\section{Discussion}

TFA is a plant-derived compound with antioxidant and antimicrobial properties [18]. A recent study showed that TFA could reduce oxidative stress by expression of genes [19]. This research reveals that TFA niosome was successfully obtained by film hydration method. The optimized formulation contained TFA $(\sim 7.72 \times 10-2 \mathrm{mmol})$, Span 60 with $(85.29 \mathrm{mg})$, Tween $60(214.71 \mathrm{mg})$, and cholesterol (95.54 mg) showed appropriate physicochemical features. The HLB of the optimized formulation was 12 . The SEM images showed that the optimized niosomal formulation has semi-spherical morphology with an average size of $158.7 \mathrm{~nm}$ (Fig. 1). The release data for both niosomal formulation and drug suspension were best fitted to the Korsmeyer-Peppas model. According to the data reported in Table 2, the $n$ value was 0.479 and 0.477 for niosomal formulation and drug suspension, respectively. The $n$ value showed that the dominated mechanism of drug release was diffusion as the $n$ value is close to 0.45. Diffusional exponent ( $n$ ) between 0.45 and 0.89 indicates non-Fickian transport release mechanism [20, 21]. As mentioned previously, the addition of the cholesterol in the formulation acts as a stabilizer and decreases the leakage [22]. The slow-release pattern of entrapped TFA may be due to the high stability of the niosomal formulation compared to the drug suspension. Apart from cholesterol, the presence of binary surfactant namely Span 60 and Tween 60 could be a reason for the better stability of noisome and slow-release pattern [22] (Fig. 2).

The MTT assay was utilized for analyzing the cytotoxicity of TFA and TFA niosome. MTT powder is a water-soluble yellow tetrazolium salt which gets into a redox reaction by the existence of the mitochondrial succinate dehydrogenase enzyme in the living cells and gets into a transformation of water-insoluble formazan dye. For spectrophotometric analysis, the insoluble purple formazan should be dissolved into a colored solution $[9,23]$. The results revealed that TFA niosome was more effective than TFA alone in the concentration of $50 \mu \mathrm{M}$ in the L929 cell line. In the cytoprotective study, HFF cells were exposed to $300 \mu \mathrm{M}$ of $\mathrm{H}_{2} \mathrm{O}_{2}$ as an oxidative stress inducer, and then, TFA niosome and TFA were added. The effect of the formulation and the pure drug to reverse the oxidative damages was measured through MTT assay. The results showed that only when $25 \mu \mathrm{M}$ the niosomal formulation was used had more cytoprotective effect than the pure drug. The antioxidant effect of TFA and TFA niosome was analyzed with an

Table 3 Minimum inhibitory concentration value $(\mu \mathrm{g} / \mathrm{mL})$ of TFA and niosomal formulation against bacteria

\begin{tabular}{lccccccc}
\hline Bacteria strain & S. aureus & E. coli & E. facelailis & P. aeruginosa & A. baumannii & K. pneumonia & P. mirabilis \\
\hline Ciprofloxacin & 0.21 & 0.1 & 0.21 & 0.25 & 0.25 & 0.1 & 0.1 \\
MIC of the drug & 750 & 750 & $>750$ & $>750$ & $>750$ & $>750$ & $>750$ \\
MBC of the drug & $>2000$ & $>2000$ & $>2000$ & $>2000$ & $>2000$ & $>2000$ & $>2000$ \\
MIC of the formulation & 375 & 187.5 & 750 & 375 & 375 & 375 & 750 \\
MBC of the formulation & 1500 & $562 / 5$ & $>2000$ & $>2000$ & 1125 & $>2000$ \\
\hline
\end{tabular}

$M I C$ minimum inhibitory concentration, $M B C$ minimum bactericidal concentration 
Fig. 6 Cytoprotection against $\mathrm{H}_{2} \mathrm{O}_{2}$-induced oxidative stress

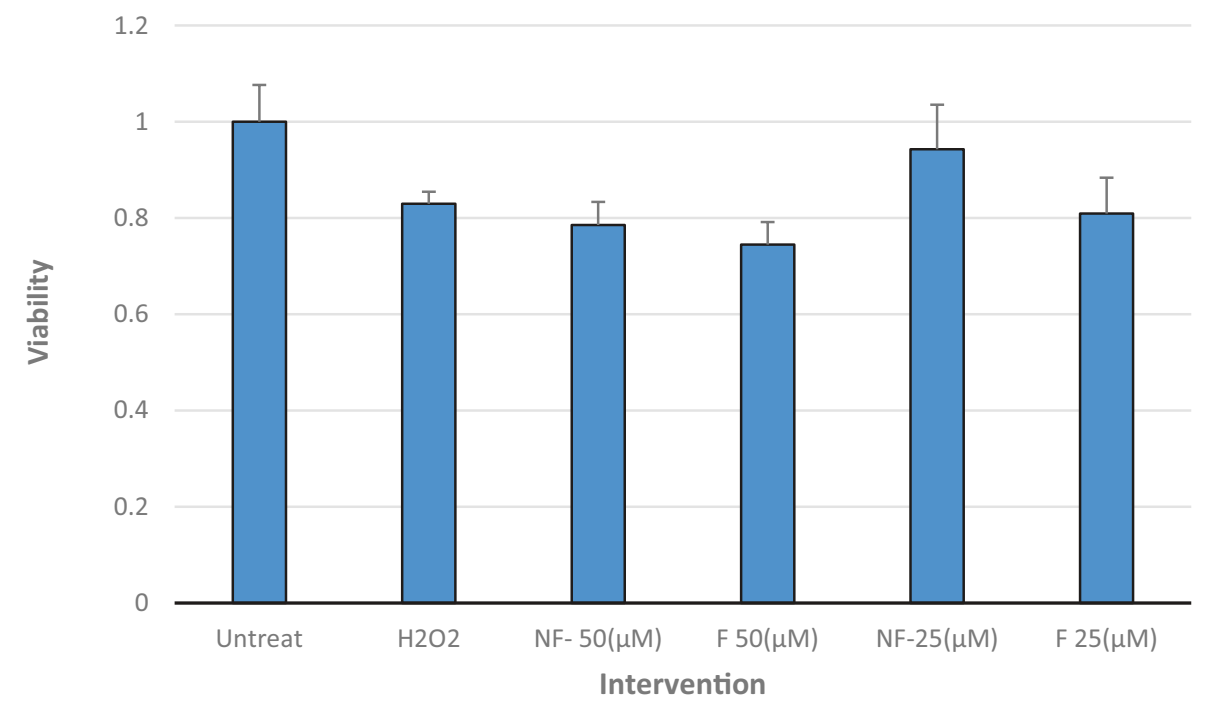

indicator called DCFH-DA which is commonly used to evaluate oxidative stress in cells. The results indicated that the decreased ROS production after $24 \mathrm{~h}$ of treatment of HFF cells with TFA niosomes and TFA at concentrations of 25 and $50 \mu \mathrm{M}$ is visible. The reduction of peroxide ions has been identified based on evidence of uptake of ROS-sensitive fluorescence markers called DCFDH. Cells treated with niosomes containing TFA at a concentration of $25 \mu \mathrm{M}$ had lower fluorescence than the control group $\left(\mathrm{H}_{2} \mathrm{O}_{2}\right.$ treatment), which was statistically significant $(P<0.05)$. Oxidative stress is a situation in which the balance of the ROS and the biological system which repairs the damage is destroyed [9]. To induce the same situation of oxidative stress, hydrogen peroxide $\left(\mathrm{H}_{2} \mathrm{O}_{2}\right)$ can be used for in vivo and in vitro. This compound has the capability of inducing oxidative damages by providing lipid peroxidation, ROS production, reducing the function of antioxidant enzymes such as glutathione peroxidase, superoxide dismutase, and catalase which leads to the cell death [9]. Antioxidants are chemicals with the ability to neutralize the toxic and volatile free radicals which are explained as atoms or groups of atoms with an unpaired electron, these radicals are toxic, volatile, and very reactive [5]. The molecular structure of phenolic acids, the presence of aromatic ring, and/or double bonds play an important role in their function as antioxidants [24]. More specifically, the unsaturated bonds in the side chain of the structure are suitable sites for free radicals to attack. The protection against lipid peroxidation can be attributed to the presence of carboxylic group which cause an interaction with the lipid bilayer of the cell membrane $[1,3]$.

There has been a relation between the etiology of more than a hundred of human diseases and the process of ageing with oxidative stress which cause cellular damages. The beneficial effects of dietary antioxidants mostly act as a protective way towards cellular damages caused by ROS. It is crucial to note that strong antioxidants may also act as prooxidants with the capability of inducing oxidative stress and cellular destruction. The antioxidant/pro-oxidant features of polyphenols are mainly depended on the oxidative condition in cells, and it is essential to know to decide their effect on cells, which can either be useful or harmful. Different perspectives on the antioxidant/pro-oxidant activity of phenolic acids could be seen as a result of the interaction of phenolic parts with ROS in separate phases. In a recent study, gallic acid and epigallocatechin gallate are known to act as potent pro-oxidants due to the oxidation of $\mathrm{Fe}^{+3}$ to $\mathrm{Fe}^{+2}$. The results showed that TFA niosomal formulation could be used as a good antioxidant, and it has the potential to scavenge free radical species.

Many researchers have reported that the niosomes with their bilayer structure could have an interaction with the outer membrane of the microorganism which leads to the better permeability of the drug into the organisms [25].In the previous study, the niosomal structure of ciprofloxacin has been prepared and the antimicrobial effect has been evaluated which showed that the bilayer structure enhanced its antimicrobial effect $[26,27]$. In another study, the niosomal formulation of moxifloxacin also has exhibited better antibacterial effect [28]. These findings suggest that the encapsulation of TFA may enhance its antibacterial effect against bacterial strains [7]. A similar pattern has been reported for niosomes containing cephalexin [29]. Cholesterol is a highly negatively charged compound and increases the chances of attaching nano-vesicles to Gramnegative bacterial cells. Furthermore, for Gram-positive bacteria, the fusogenic characteristic of cholesterol is not useful [26], due to the peptidoglycan barrier which in this 
case the peptidoglycan layer of Gram-positive bacteria can have interactions with niosomes so the drug will be released through the bacteria's cell [27].

\section{Conclusion}

The following research revealed the antioxidant and antibacterial effects of TFA niosomes. This research displayed that, the optimized formulation could be suitable for scavenging free radical species; in addition, they have a marked capability of protecting human foreskin fibroblasts in vitro from oxidative stress induced by hydrogen peroxide. TFA niosome was released in a more controlled manner than the drug dispersion. The research showed that the optimized niosomal formulation containing TFA could decrease the minimum inhibitory concentration of the drug.

Funding The Mazandaran University of Medical Science financially supported this study with a grant number of 134 .

\section{Declarations}

Conflict of Interest The authors declare that they have no conflict of interest.

Open Access This article is licensed under a Creative Commons Attribution 4.0 International License, which permits use, sharing, adaptation, distribution and reproduction in any medium or format, as long as you give appropriate credit to the original author(s) and the source, provide a link to the Creative Commons licence, and indicate if changes were made. The images or other third party material in this article are included in the article's Creative Commons licence, unless indicated otherwise in a credit line to the material. If material is not included in the article's Creative Commons licence and your intended use is not permitted by statutory regulation or exceeds the permitted use, you will need to obtain permission directly from the copyright holder. To view a copy of this licence, visit http://creativecommons.org/licenses/by/4.0/.

\section{References}

1. Srinivasan M, Sudheer AR, Menon VP. Ferulic acid: therapeutic potential through its antioxidant property. J Clin Biochem Nutr. 2007;40(2):92-100. https://doi.org/10.3164/jcbn.40.92.

2. Doss HM, Samarpita S, Ganesan R, Rasool M. Ferulic acid, a dietary polyphenol suppresses osteoclast differentiation and bone erosion via the inhibition of RANKL dependent NF- $\kappa \mathrm{B}$ signalling pathway. J Life Sci. 2018;207:284-95. https://doi. org/10.1016/j.1fs.2018.06.013.

3. Itagaki S, Kurokawa T, Nakata C, Saito Y, Oikawa S, Kobayashi $\mathrm{M}$, et al. In vitro and in vivo antioxidant properties of ferulic acid: a comparative study with other natural oxidation inhibitors. Food Chem. 2009;114(2):466-71. https://doi. org/10.1016/j.foodchem.2008.09.073.
4. Jo ER, Youn CK, Jun Y, Cho SI. The protective role of ferulic acid against cisplatin-induced ototoxicity. Int J Pediatr Otorhinolaryngol. 2019;120:30-5. https://doi.org/10.1016/j. ijporl.2019.02.001.

5. Maurya DK, Devasagayam TPA. Antioxidant and prooxidant nature of hydroxycinnamic acid derivatives ferulic and caffeic acids. Food Chem Toxicol. 2010;48(12):3369-73. https://doi. org/10.1016/j.fct.2010.09.006.

6. Itagaki S, Kurokawa T, Nakata C, Saito Y, Oikawa S, Kobayashi $\mathrm{M}$, Hirano T, Iseki K. In vitro and in vivo antioxidant properties of ferulic acid: a comparative study with other natural oxidation inhibitors. Food Chem. 2009;114(2):466-71. https://doi. org/10.1016/j.foodchem.2008.09.073.

7. Sundaramoorthy NS, Mitra K, Ganesh JS, Makala H, Lotha R, Bhanuvalli SR, et al. Ferulic acid derivative inhibits NorA efflux and in combination with ciprofloxacin curtails growth of MRSA in vitro and in vivo. Microb Pathog. 2018;124:54-62. https:// doi.org/10.1016/j.micpath.2018.08.022.

8. Chen S, Hanning S, Falconer J, Locke M, Wen J. Recent advances in non-ionic surfactant vesicles (niosomes): Fabrication, characterization, pharmaceutical and cosmetic applications. Eur J Pharm Biopharm. 2019;144:18-39. https:// doi.org/10.1016/j.ejpb.2019.08.015.

9. Nadal JM, Gomes MLS, Borsato DM, Almeida MA, Barboza FM, Zawadzki SF, et al. Spray-dried Eudragit ${ }^{\circledR}$ L100 microparticles containing ferulic acid: formulation, in vitro cytoprotection and in vivo anti-platelet effect. Mater Sci Eng C. 2016;64:318-28. https://doi.org/10.1016/j.msec.2016.03.086.

10. Rezaeiroshan A, Saeedi M, Morteza-Semnani K, Akbari J, Gahsemi M, Nokhodchi A. Development of trans-Ferulic acid niosome: an optimization and an in-vivo study. J Drug Deliv Sci Technol. 2020;59:101854. https://doi.org/10.1016/j.jddst.2020.101854.

11. Do Nascimento TG, Da Silva PF, Azevedo LF, Da Rocha LG, de Moraes Porto ICC, e Moura TFAL, et al. Polymeric nanoparticles of Brazilian red propolis extract: preparation, characterization, antioxidant and leishmanicidal activity. Nanoscale Res Lett. 2016;11(1):301. https://doi.org/10.1186/ s11671-016-1517-3.

12. Zhang Y, Li Z, Zhang K, Yang G, Wang Z, Zhao J, et al. Ethyl oleate-containing nanostructured lipid carriers improve oral bioavailability of trans-ferulic acid as compared with conventional solid lipid nanoparticles. Int J Pharm. 2016;511(1):57-64. https:// doi.org/10.1016/j.ijpharm.2016.06.131.

13. Manca ML, Marongiu F, Castangia I, Catalán-Latorre A, Caddeo C, Bacchetta G, et al. Protective effect of grape extract phospholipid vesicles against oxidative stress skin damages. Ind Crops Prod. 2016;83:561-7. https://doi.org/10.1016/j.indcrop.2015.12.069.

14. Manca ML, Castangia I, Caddeo C, Pando D, Escribano E, Valenti D, et al. Improvement of quercetin protective effect against oxidative stress skin damages by incorporation in nanovesicles. Colloid Surface B. 2014;123:566-74. https://doi. org/10.1016/j.colsurfb.2014.09.059.

15. Bonini MG, Rota C, Tomasi A, Mason RP. The oxidation of 2', 7'-dichlorofluorescin to reactive oxygen species: a self-fulfilling prophesy? Free Radic Biol Med. 2006;40(6):968-75. https://doi. org/10.1016/j.freeradbiomed.2005.10.042.

16. Wikler MA. Methods for dilution antimicrobial susceptibility tests for bacteria that grow aerobically: approved standard. CLSI (NCCLS). 2006;26:M7-A7.

17. Dwivedi A, Mazumder A, Nasongkla N. Layer-by-layer nanocoating of antibacterial niosome on orthopedic implant. Int J Pharm. 2018;547(1-2):235-43. https://doi.org/10.1016/j. ijpharm.2018.05.075.

18. Doss HM, Dey C, Sudandiradoss C, Rasool MK. Targeting inflammatory mediators with ferulic acid, a dietary polyphenol, 
for the suppression of monosodium urate crystal-induced inflammation in rats. Life Sci. 2016;148:201-10. https://doi. org/10.1016/j.1fs.2016.02.004.

19. Guvvala PR, Ravindra JP, Selvaraju S, Arangasamy A, Venkata KM. Ellagic and ferulic acids protect arsenic-induced male reproductive toxicity via regulating Nfe212, Ppargc1a and StAR expressions in testis. Toxicology. 2019;413:1-12. https://doi. org/10.1016/j.tox.2018.11.012.

20. Ritger PL, Peppas NA. A simple equation for description of solute release I Fickian and non-fickian release from nonswellable devices in the form of slabs, spheres, cylinders or discs. J Control Release. 1987;5(1):23-36. https://doi. org/10.1016/0168-3659(87)90034-4.

21. Farmoudeh A, Rezaeiroshan A, Abbaspour M, Nokhodchi A, Ebrahimnejad P. Solid dispersion pellets: an efficient pharmaceutical approach to enrich the solubility and dissolution rate of deferasirox. Biomed Res Int 2020. 2020. https://doi. org/10.1155/2020/8583540.

22. Basiri L, Rajabzadeh G, Bostan A. Physicochemical properties and release behavior of Span 60/Tween 60 niosomes as vehicle for $\alpha$-Tocopherol delivery. LWT. 2017;84:471-8. https://doi. org/10.1016/j.lwt.2017.06.009.

23. Tavano L, Vivacqua M, Carito V, Muzzalupo R, Caroleo MC, Nicoletta F. Doxorubicin loaded magneto-niosomes for targeted drug delivery. Colloid Surface B. 2013;102:803-7. https://doi. org/10.1016/j.colsurfb.2012.09.019.

24. Moriguchi N, Hinoi E, Tsuchihashi Y, Fujimori S, Iemata M, Takarada $\mathrm{T}$, et al. Cytoprotection by pyruvate through an antioxidative mechanism in cultured rat calvarial osteoblasts. Histol Histopathol. 2006. https://doi.org/10.14670/HH-21.969.
25. Barakat HS, Kassem MA, El-Khordagui LK, Khalafallah NM. Vancomycin-eluting niosomes: a new approach to the inhibition of staphylococcal biofilm on abiotic surfaces. AAPS PharmSciTech. 2014;15(5):1263-74. https://doi.org/10.1208/ s12249-014-0141-8.

26. Moazeni E, Gilani K, Sotoudegan F, Pardakhty A, Najafabadi $\mathrm{AR}$, Ghalandari R, et al. Formulation and in vitro evaluation of ciprofloxacin containing niosomes for pulmonary delivery. J Microencapsul. 2010;27(7):618-27. https://doi.org/10.3109/ 02652048.2010 .506579$.

27. Akbari V, Abedi D, Pardakhty A, Sadeghi-Aliabadi H. Ciprofloxacin nano-niosomes for targeting intracellular infections: an in vitro evaluation. J Nanopart Res. 2013;15(4):1556. https://doi.org/10.1007/s11051-013-1556-y.

28. Sohrabi S, Haeri A, Mahboubi A, Mortazavi A, Dadashzadeh S. Chitosan gel-embedded moxifloxacin niosomes: an efficient antimicrobial hybrid system for burn infection. Int J Biol Macromol. 2016;85:625-33. https://doi.org/10.1016/j.ijbiomac.2016.01.013.

29. Ghafelehbashi R, Akbarzadeh I, Yaraki MT, Lajevardi A, Fatemizadeh M, Saremi LH. Preparation, physicochemical properties, in vitro evaluation and release behavior of cephalexin-loaded niosomes. Int J Pharm. 2019;569:118580. https://doi.org/10.1016/j. ijpharm.2019.118580.

Publisher's Note Springer Nature remains neutral with regard to jurisdictional claims in published maps and institutional affiliations. 